\title{
The possibilities of using Bluetooth to control the lift platform used for people with reduced mobility
}

\author{
Milan Adamek ${ }^{1,{ }^{*}}$, Martin Reznicek ${ }^{1}$, Martin Pospisilik ${ }^{1}$, Petr Neumann ${ }^{1}$ \\ ${ }^{1}$ Tomas Bata University in Zlín, Faculty of Applied Informatics, Nam. T. G. Masaryka 5555, 76005 Zlín, Czech Republic
}

\begin{abstract}
The paper presents the design of a control unit and an application for Android OS, which will use the Bluetooth transmission to control vertical lifting devices for people with reduced mobility. The theoretical part consists of the description of the Android Studio development environment, the description of data transfer using Bluetooth, and the estimation of the reduction in platform production costs and the competitiveness of these products on the global market. From the theoretical part there is a practical part, which deals with the development of a new control unit for lifting equipment ZP5, using mobile application and introducing these technologies into the production process. The practical part also describes the effect of these adjustments on increasing the production and sale of vertical staircase platforms with a view to use for other products for people with reduced mobility.
\end{abstract}

\section{Introduction}

In parallel with the aging of the European population, the number of persons with reduced mobility is set at 80 million (in the EU). Our society needs the economic, social and cultural potential of people with limited abilities and legislation. [1]

In existing buildings, it is often available how barrierfree access is available through a built-in staircase, using a type of platform, jack or seat, tailored to the conditions. There are many types of inclined lifting and vertical lifting-stair platforms for immobile, stair seats for pedestrians and walkways, load lifting systems for home and professional use.

This article deals with the mapping of the possibilities of creating a custom-built drone capable of recording an image and assessing its suitability for use in the 3D visualization of an object, with the selected software. The practical part demonstrates the use of these assemblies on a selected object.

\section{Lifting platform types}

\subsection{Slanted stair platforms}

Generally, electrically driven and controlled platforms move along a double guide rail, which can be anchored to a wall, stair steps, or self-supporting. They are equipped with raids and doors or a protective bar that opens in the station. They can be used on standard, atypical, spiral and combined staircases and have small space requirements. It is used to transport immobile persons (including or without a wheelchair) between the individual floors of a building by a staircase. [4]

\footnotetext{
* Corresponding author: adamek@fai.utb.cz
}

\subsection{Stair seats}

Stair seats are used to transport immobile persons without a wheelchair between the individual floors of a building by a staircase. Like stair platforms, they are guided along a double rail anchored to a wall or staircase. [2]

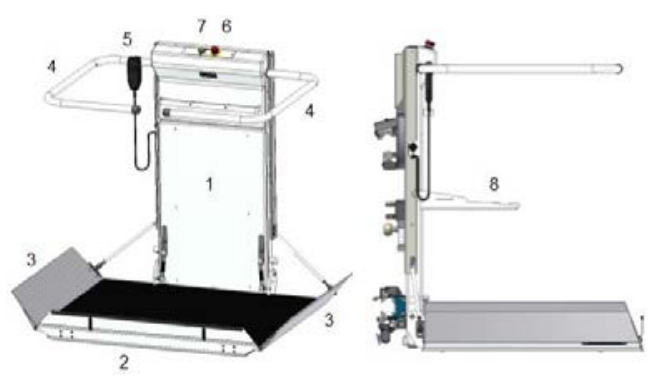

Fig. 1. Sloping staircase platform [3]

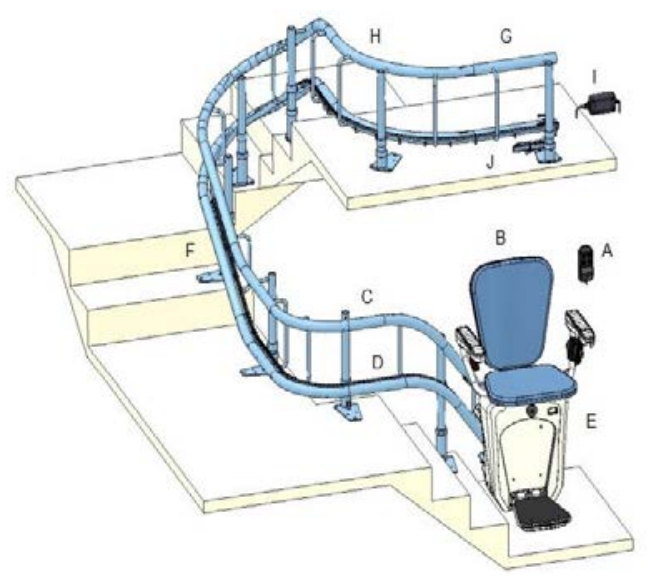


Fig. 2. SA-ALFA sloping staircase seat [4]

\subsection{Vertical stair platforms}

Vertical or vertical lift stair platforms for persons with reduced mobility are also known as home lift or lift platform. They can be used to overcome a height difference of up to 20 meters. It is a cheaper replacement for the elevator, which can be installed both indoors and outdoors, in private homes, industrial buildings, businesses, hospitals and similar facilities. [2]

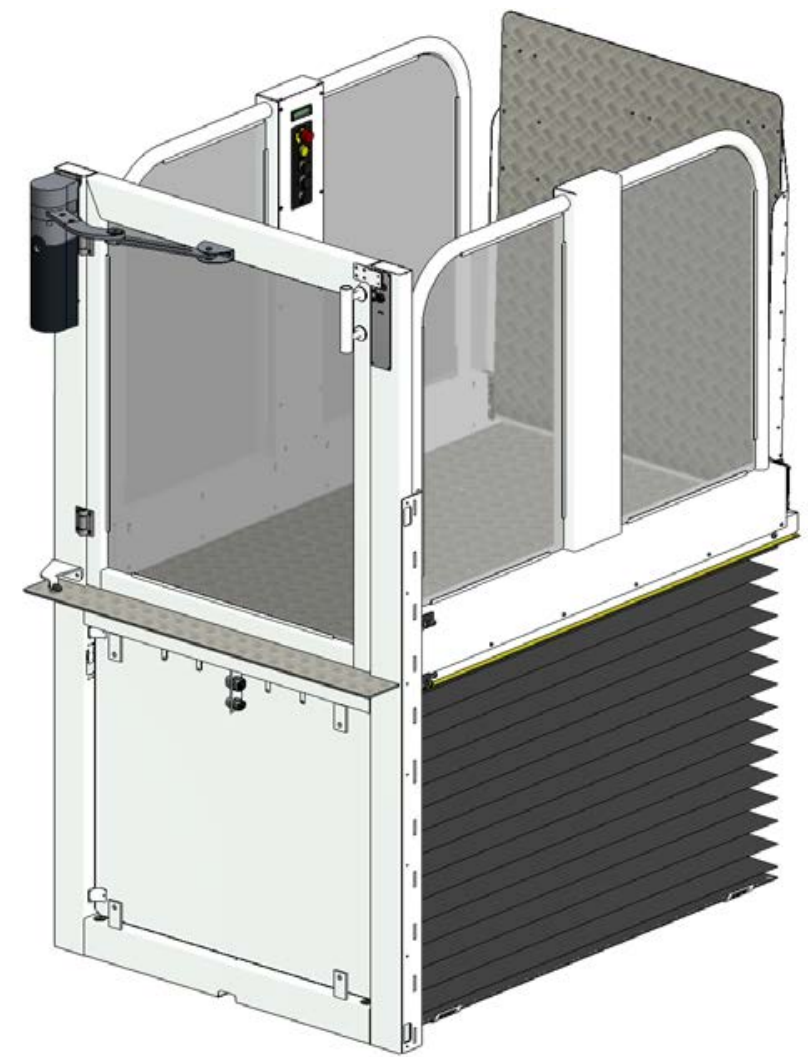

Fig. 3. Vertical stair platforms [4]

Ceiling hoists are used to move immobile or poorly moving people at home, in spas, in retirement homes or in hospitals (for example, from bed to bath, toilet, or other room) using a lifting device anchored on the ceiling or walls.

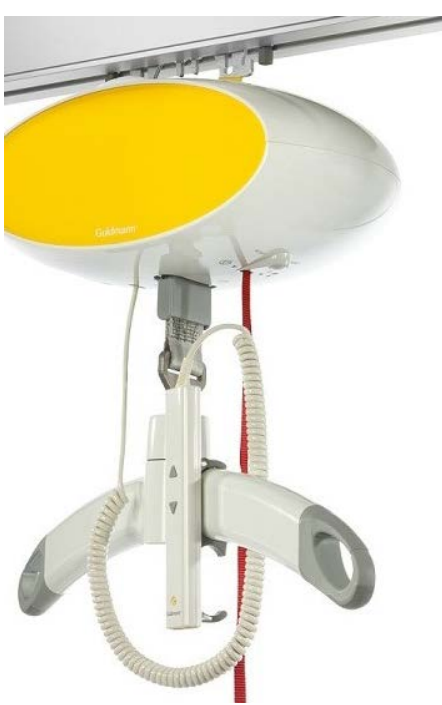

Fig. 4. Ceiling hoist GH-1 [3]

The advantage of these devices is easy handling of the assistant with the client and high user comfort thanks to the possibility to use different types of bags. Ceiling hoist systems are among the devices featuring quick and easy installation and easy maintenance. [2]

\section{Lifting platform controls}

Platform control means either a handheld controller connected to the platform by a spiral cable, or controls located directly on the platform upper cover. They are equipped with up and down direction buttons, an emergency stop button and an alarm. Using the direction buttons from the platform controls, the platform can only be operated with the (open) floor lowered. In addition to the direction of travel, these buttons also control the movement of raids and barriers when the platform stands at one of the stops.

If both travel direction buttons (up / down) on the platform control are pressed for more than 5 seconds, the menu is activated. The push-button emergency stop button blocks all platform activity. The subsequent release is possible by turning the button in the direction of the arrows.

When the emergency message button is activated, an acoustic alarm is triggered and the emergency alarm relay on the drive control board is triggered (even in the event of a power failure). 


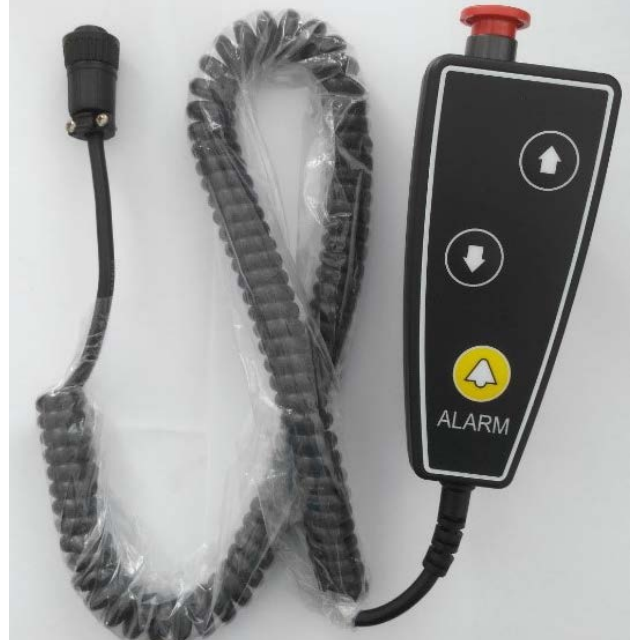

Fig. 5. Platform driver Kingstec

\subsection{Radio Controls for Platforms}

In each radio-controlled platform, a Schmidiger receiver is installed, which can work with T20 or T60 transmitters. T20TX-04WEI transmitters are fitted as standard in the wall-mounted controls. If more than 10 transmitters (wall controllers) are assigned, they are initially erased as the first programmed transmitter. It is not possible to delete an already programmed transmitter. However, it is possible to delete all already programmed transmitters at the same time. The receiver then does not respond to any signal until at least one transmitter is programmed. The receiver can be programmed with any transmitter button (wall controller).

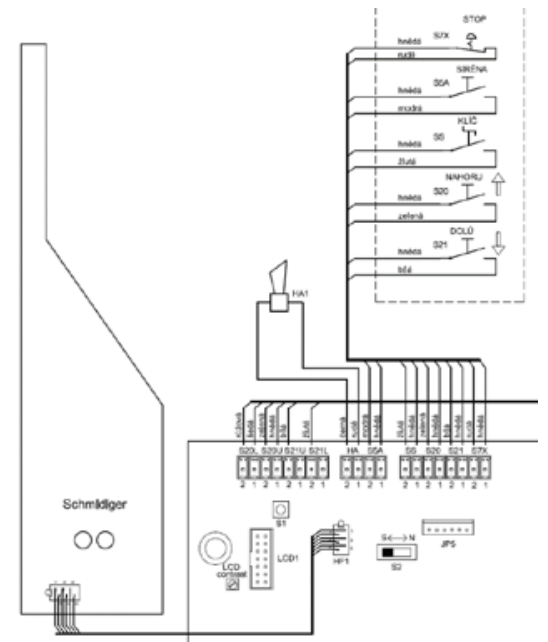

Fig. 6. Schematic connection of Schimidiger receiver with control unit ZP5

\section{Design of platform control using a mobile device}

Bluetooth wireless technology, which is part of almost every mobile device available today, is used to connect the platform or its control unit and mobile device. The Bluetooth interface is implemented into the platform controller via a Bluetooth module. An application for controlling the direction of movement of the platform will be created for the mobile device.

\subsection{Bluetooth}

Bluetooth communication has been developed to replace cables between your computer and remote devices such as printers and other peripherals. Today, the Bluetooth standard is mainly used in mobile phones for data transmission, for communication between consumer electronics and smart homes. [5]

Bluetooth technology consists of two parts - hardware and software. As far as hardware is concerned, Bluetooth has been deliberately designed, as simple as possible with a minimum of analog high-frequency components and without bulky and expensive components in order to achieve the lowest possible cost and thereby support its expansion. Basically it consists of three basic components: a Bluetooth radio that acts as both a transmitter and receiver, a Bluetooth Link Controller that controls connection establishment and communication, identification and access. The third component is the Bluetooth Link Manager, which prepares data and ensures communication between devices equipped with a Bluetooth module. [6]

\subsection{Bluetooth topology}

Bluetooth technology supports two types of communication - point and multipoint. In the case of point-to-point transmission it is a classic point to point communication, direct connection of two devices. In the case of multi-point, so-called multi-point communication, one device has a status master. Thus, a device with this designation serves as an access point to which up to 7 other slave devices can be connected. This grouping is called a piconet. These picosets can be further combined into so-called scatter-net networks. There can be a maximum of 10 picosets in one spread network. Every Bluetooth device should have its name, the so-called Bluetooth Device Adrress, which is some equivalent of the MAC address of Internet protocols. [5]

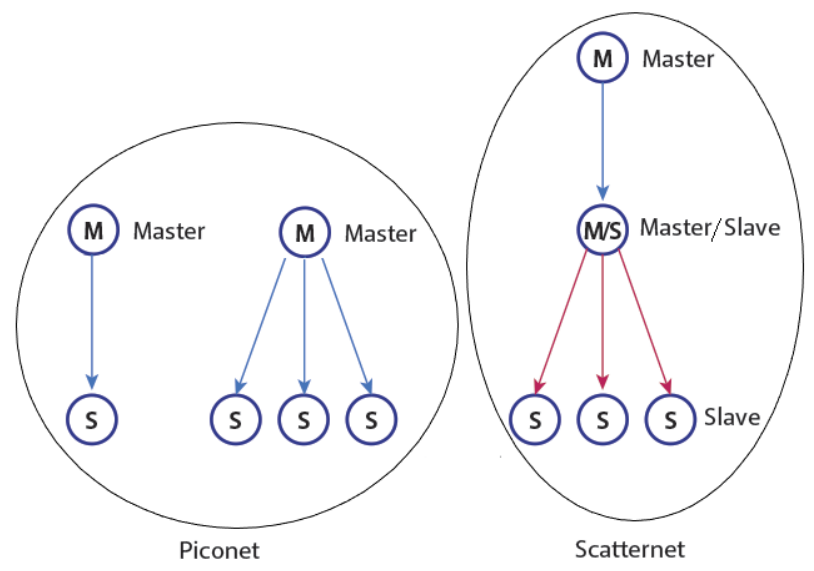

Fig. 7. Sample of piconet and scatternet networks . [6] 


\subsection{Preparing the Bluetooth module to connect to} the controller

The Bluetooth module $\mathrm{HC}-05$ is a communication module that allows you to wirelessly connect a device with a serial UART interface (Universal Asynchronous Receiver / Transmitter) to another Bluetooth enabled device. The module is based on Bluetooth technology in version 2.0, then communicates with the device via serial line. The communication range is limited to a distance of about 10 meters in the open air.
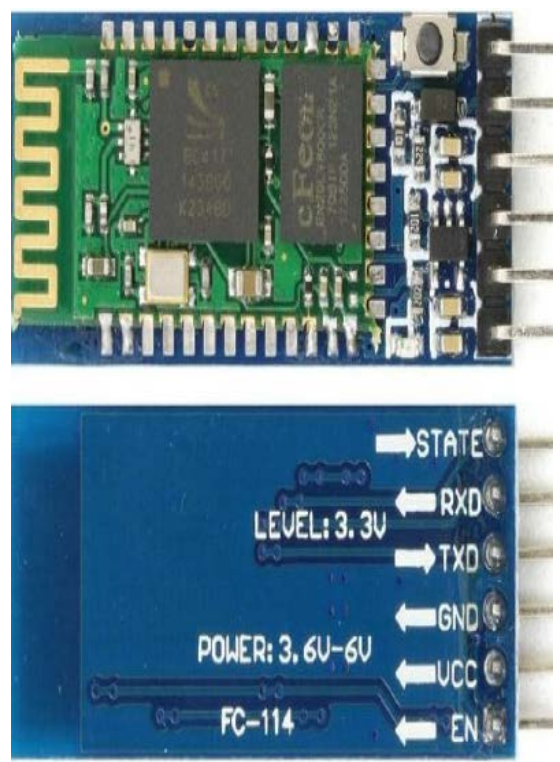

Fig. 8. Bluetooth modul HC-05

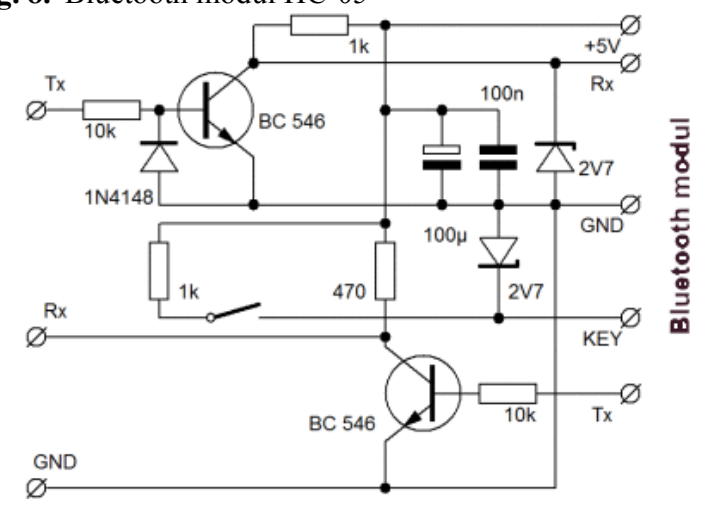

Fig. 9. Bluetooth module configuration tool

The HC-05 requires a supply voltage of between 3.3 and 6 VSS. With a 5 VSS supply, the current consumption is about $2 \mathrm{~mA}$ at standstill and reaches a maximum of $40 \mathrm{~mA}$ during communication. It should be noted that, despite the above-mentioned voltage range, the input and output levels of the logic signal should be at most +3.3 VSS.

\subsection{Connecting the configured module to the platform control unit}

The platform control unit includes a PIC 16LF1509 microprocessor that has a directly implemented UART serial port - pin 10 (RB7 / TX) used for transmission, and pin 12 (RB5 / AN11 / RX) is used to receive serial communication. At the same time, this interface is used for communication with the radio module Schmidiger connected via a connector marked JP1.

Instead of the radio module, the configured $\mathrm{HC}-05$ Bluetooth module is connected to the JP1 connector, it is necessary to interconnect the TX, RX signals and connect the +5 VSS power supply and connect the module and controller countries. The location of the Bluetooth module in the platform control unit is shown in Figure 11.

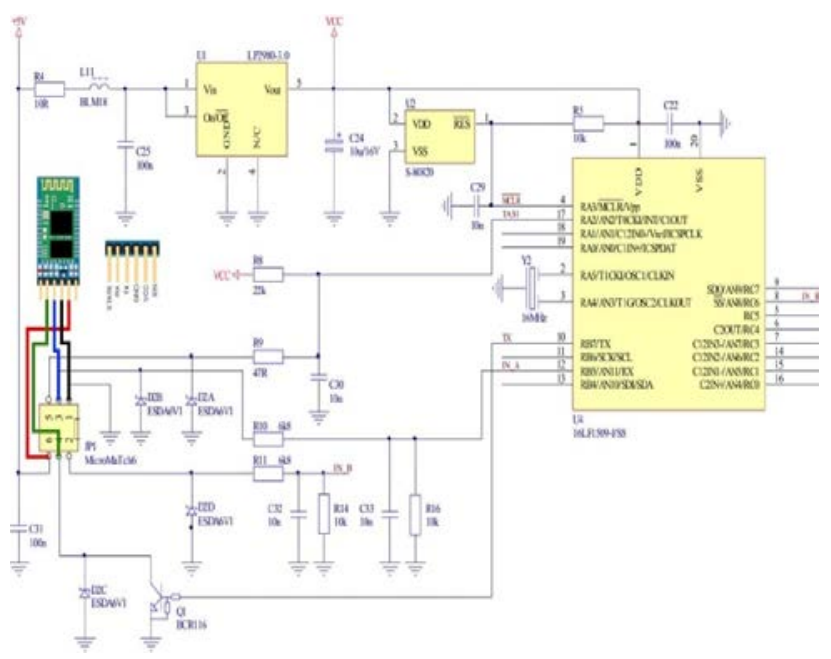

Fig. 10. Connecting the Bluetooth Module to the Platform Control Module

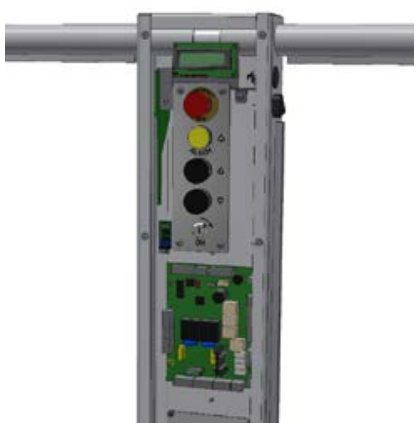

Fig. 11. Locating the Bluetooth module in the ZP5 platform control unit

\subsection{Communication protocol}

When connecting the Bluetooth module to the platform control unit, it is necessary to respect not only the hardware connection but also the UART communication protocol itself, which is set in the unit as follows:

- communication speed... $2400 \mathrm{Bd}$,

- transferring ... 8 bits

- No parity

- no handshake. 
The controller controller expects two bytes at 2400 msec / $2400 \mathrm{bit} / \mathrm{s}$ every $100 \mathrm{~ms}$ to define the required platform command. An overview of defined platform commands is given in Tab. 1 .

Table. 1. Overview of defined platform commands

\begin{tabular}{|l|l|}
\hline $\begin{array}{l}\text { Command } \\
\text { (hexadecimal) }\end{array}$ & $\begin{array}{l}\text { Description / meaning of the } \\
\text { command }\end{array}$ \\
\hline 04 FB & Move the platform up \\
\hline 08 F7 & Platform Movement Down \\
\hline 01 FE & Open platform guard \\
\hline 02 FD & Closing the platform guard \\
\hline
\end{tabular}

\subsection{Mobile application}

The mobile platform control application was primarily created for the Android operating system, which is currently the most widespread $(>85 \%)$ and at the same time the fastest growing mobile operating system.

When developing a mobile application, a free-to-use Android Studio development environment was used directly from Google, which is also the creator of the Android operating system itself. The resulting mobile application is written in object-oriented programming language JAVA. A number of integrated software libraries were used, especially the BluetoothSocket used to operate the mobile phone / tablet Bluetooth interface. The source code of the created ALTECH APP application is included in the enclosed CD.

The native parts of Android Studio are:

- Android Studio IDE itself

- Android SDK Tools

- Android compiler

- Basic emulators with full Android.

Android Studio also features a sophisticated editor with the ability to design a user interface using drag-and-drop - the app's design was designed in design mode and added in XML markup language (eXtensible Markup Language). The application was debugged in the integrated Nexus 10 mobile emulator.

The application was compiled for the latest version of Android, so it should not be a problem with its compatibility with mobile devices with the latest Android operating system version 8.0 (OREO) - the result is an executable installation package ALTECH.apk, which can be used to install the mobile application on your mobile device with Android.

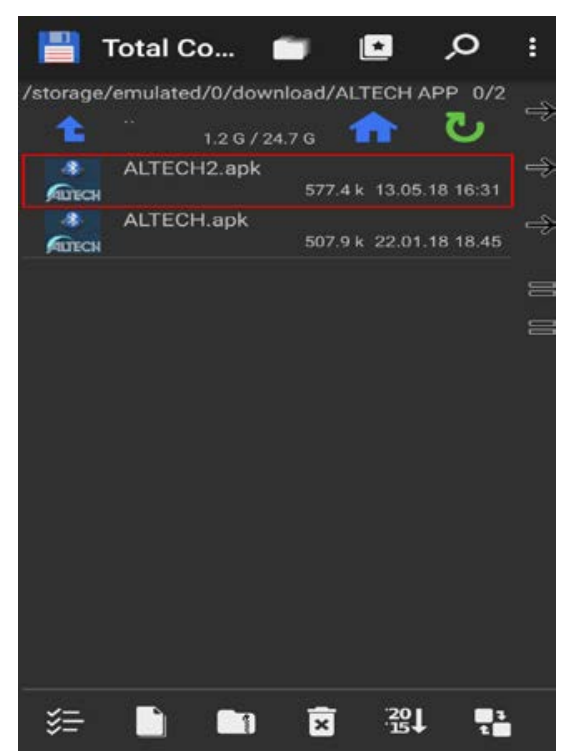

Fig. 12. ALTECH APP Application - Installation Files

After installing the app on an Android mobile phone or tablet, an executable application shortcut will be created on the device desktop along with two so-called widgets that can be used to directly control platform movement without launching the application.

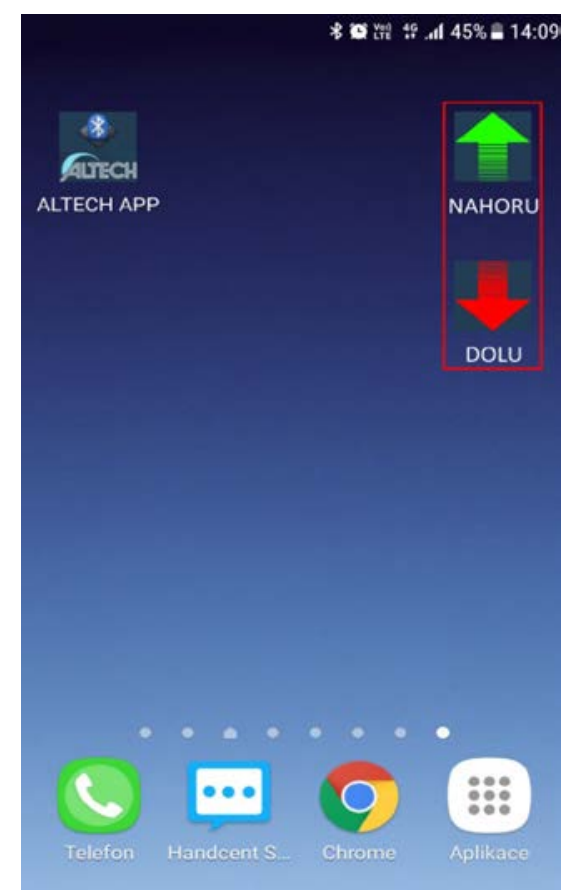

Fig. 13. Applications ALTECH APP - link and widgets

The ALTECH.apk application was created as a trial version of the application, which was primarily intended to debug the correct communication of the mobile device with the platform control unit. ALTECH2.apk is then the installation package of the final version of the application.

The purpose of ALTECH APP itself is to provide the user with the most user-friendly and simple way to operate the platform with mobile devices, so the application contains only two buttons to determine the direction of motion of the platform without the possibility of further adjustment. By doing so, the fear of using 
similar technology should be minimized, especially for seniors.

To ease the use of the application, feedback on button presses has been implemented, whereby the user is informed by a different vibration of the mobile device, along with an acoustic signal, which button or direction of movement of the platform has been triggered.

\section{Conclusion}

In addition to the successful verification of the implemented solution (controlling the movement of the platform using the created mobile application) in practice, a measurement was performed using the logical analyzer SALEAE LOGIC16, which uses a personal computer with the SALEAE LOGIC application installed to process and display the measured digital signals. The application can also decode the measured signal and directly display the sequence of individual commands sent from the ALTECH APP mobile application to the platform control unit. The individual signals, respectively commands for the platform, were directly measured on the controller pin of the controller, dedicated for serial communication with the Bluetooth module.

To move the platform downwards, the mobile application should send the HEX (08 F7) command periodically every $100 \mathrm{~ms}$ while the DOWN button is pressed. Measured waveforms of the control signal sent from the Bluetooth module to the platform controller demonstrate the correctness of the solution.

This work was supported by the Ministry of Education, Youth and Sports of the Czech Republic within the National Sustainability Programme project No. LO1303 (MSMT-7778/2014) and also by the European Regional Development Fund under the project CEBIA-Tech No. CZ.1.05/2.1.00/03.0089.

\section{References}

1. EN 81-40: Safety regulations for the construction and installation of lifts - Special lifts for the transport of persons and passengers - Vertical lifting platforms for the transport of persons with reduced mobility (2009).

2. Products. Altech.cz [online]. [cit.. 2018-04-16]. https://www.altech.cz/produkty/

3. Altech, spol. s.o. OMEGA Technical Guide Documentation. Uherske Hradiste, 32 p, (2015).

4. Altech, spol. s.o. ALFA Technical Guide Documentation. Uherske Hradiste, 32 p, (2015).

5. Puzmova, R. Wireless communication security: how to secure Wi-Fi, Bluetooth, GPRS or 3G. Brno, ISBN 80-251-0791-4, (2005)
6. Gerhmann, Ch, Person J., Smeets B. Bluetooth security. Boston. ISBN 1580538851. (2004) 